\title{
Dynamics of droplets moving on a solid surface: Lattice Boltzmann simulations
}

\author{
Alexander Kupershtokh $h^{1,2, a}$ \\ ${ }^{1}$ Lavrentyev Institute of Hydrodynamics SB RAS, Acad. Lavrentyev ave., 15, Novosibirsk, 630090, Russia \\ ${ }^{2}$ National Research Novosibirsk State University, Pirogova Str., 2, Novosibirsk, 630090, Russia
}

\begin{abstract}
The three-dimensional dynamics of droplets on inclined and vertical walls in a gravity field is simulated. The dependence of a contact angle on a velocity of contact line is obtained for slow regime and smooth surface. The simulations of a rupture of the liquid films on the nonwettable solid substrate due to the thermocapillary effect (Marangoni effect) are also carried out. The lattice Boltzmann method is successfully implemented for computer simulation of these three-dimensional problems.
\end{abstract}

\section{Introduction}

The understanding of the behaviour of thin liquid films and droplets placed on the surface of solid substrates is very important for modern technologies.

A static contact angle is closely related with forces of interaction of molecules of fluid and a solid substrate. Even for sessile and pendant drops on inclined surfaces, a contact angle depends on an azimuthal angle and changes from the advancing point to the receding point. In general case, the contact angle depends on a local curvature radius of the contact line. For the stable shape of 3D static drop on an inclined surface, the Young-Laplace equation is valid

$$
2 \sigma \kappa_{\mathrm{m}}=\Delta p_{*}+\left(\rho_{\text {ж }}-\rho_{\Gamma}\right)(\mathbf{g} \cdot \mathbf{z}+\mathbf{g} \cdot \mathbf{x}),
$$

where $\sigma$ is the surface tension of liquid, $\kappa_{\mathrm{m}}=\left(\kappa_{1}+\kappa_{2}\right) / 2$ is the mean curvature of the liquid-air interface, $\mathbf{z}=\mathbf{n} z, \mathbf{x}=\boldsymbol{\tau} x$. Here, $\mathbf{n}$ and $\boldsymbol{\tau}$ are the unit normal vector and unit tangent vector to the solid surface.

A large number of experiments showed that for motion of contact line along the horizontal solid substrate, the advancing contact angle is greater than the receding angle (hysteresis). In general case, the contact angles depend also on the velocity of a contact line. Moreover, in several works, the phenomenon of dynamic contact angles and a singularity at a contact line were investigated.

A non-stationary motion of a drop can only be simulated numerically. The viscous flow of fluid with surface tension of liquid and the interaction of a liquid with a solid wall should be described in computer simulations. The most important problem is the interaction forces between fluid and solid at the interface. This phenomenon can be described better using the mesoscopic methods that are closer to the nature of such interaction than macroscopic approaches.

\footnotetext{
${ }^{\text {a }}$ Corresponding author : skn@hydro.nsc.ru
} 


\section{The lattice Boltzmann method}

The lattice Boltzmann method (LBM) is successfully implemented for computer simulation of these three-dimensional problems. The lattice Boltzmann method describes the viscous flows of fluids with an arbitrary equation of state and simulates the interfaces between vapor and liquid phases with a surface tension.

The three-dimensional version of lattice Boltzmann method D3Q19 [1] with nineteen vectors $\mathbf{c}_{k}$ of pseudo-particle velocities on a cubic lattice is realized. The evolution equation for the distribution functions $N_{k}$ can be written in the form

$$
N_{k}\left(\mathbf{x}+\mathbf{c}_{k} \Delta t, t+\Delta t\right)=N_{k}(\mathbf{x}, t)+\Omega_{k}(N)+\Delta N_{k},
$$

where $\Delta t$ is the time step, $\Omega_{k}$ is the collision operator, and $\Delta N_{k}$ is the change of the distribution functions due to the action of the internal and external body forces.

The hydrodynamic variables (the density $\rho$ and the velocity $\mathbf{u}$ of fluid) in a node are calculated as

$$
\rho=\sum_{k=0}^{b} N_{k} \text { and } \rho \mathbf{u}=\sum_{k=1}^{b} \mathbf{c}_{k} N_{k} .
$$

The collision operator is usually used in Bhatnagar-Gross-Krook (BGK) form

$$
\Omega_{k}=\left(N_{k}^{e q}(\rho, \mathbf{u})-N_{k}(\mathbf{x}, t)\right) / \tau,
$$

where $\tau$ is the dimensionless relaxation time.

The Exact Difference Method (EDM) [2] is used for the implementation of the body forces (internal forces and gravity) in the LBM:

$$
\Delta N_{k}(\mathbf{x}, t)=N_{k}^{e q}(\rho, \mathbf{u}+\Delta \mathbf{u})-N_{k}^{e q}(\rho, \mathbf{u}),
$$

where the value of the velocity after the action of the total force $\mathbf{F}$ on a node is equal to $\mathbf{u}+\Delta \mathbf{u}=\mathbf{u}+\mathbf{F} \Delta t / \rho$. The corresponding equilibrium distribution functions $N_{k}^{e q}$ are calculated as

$$
N_{k}^{e q}(\rho, \mathbf{u})=\rho w_{k}\left(1+\frac{\mathbf{c}_{k} \mathbf{u}}{\theta}+\frac{\left(\mathbf{c}_{k} \mathbf{u}\right)^{2}}{2 \theta^{2}}-\frac{\mathbf{u}^{2}}{2 \theta}\right) .
$$

To simulate boundaries between liquid and gas, the internal forces acting between nodes of fluid were introduced by Shan and Chen [3]. For equation of state $P(\rho, T)$, the total force acting on a node was introduced by Qian [4] as a gradient of the pseudopotential $\mathbf{F}=-\nabla U$, where $U(\rho, T)=P(\rho, T)-\rho \theta$. The isotropic finite-difference approximation of the gradient operator was proposed by Kupershtokh et al. [5]. We use the quite simple well-known "bounce-back" rule to implement the no-slip boundary conditions at the solid walls in the LBM simulations.

The effect of solid walls on fluids is simulated by interaction forces acting on a node $\mathbf{x}$ belonging to the fluid from the nearest nodes $\mathbf{x}+\mathbf{e}_{k}$ representing the solid boundaries [6]

$$
\mathbf{F}_{k}=w_{k} \psi(\rho(\mathbf{x})) B\left(\mathbf{x}+\mathbf{e}_{k}\right) \mathbf{e}_{k} .
$$

Thus, contact angles are not prescribed in lattice Boltzmann method but are simulated in natural way.

The Graphics Processing Units (GPUs) are exploited for all computer simulations.

\section{Droplets moving on solid surface}

The three-dimensional dynamics of droplets on inclined and vertical walls in a gravity field was simulated. The results of computer simulations for the droplet on inclined substrate $\left(\alpha=40^{\circ}\right)$ are shown in Fig. 1. The important parameter for the problem is the Bond number Bo $=\rho g H^{2} / \sigma$. It represents a dimensionless parameter used to characterize the ratio of gravity force to surface tension force. Here $\rho$ is the density of liquid droplet. The Bond number in dimensionless variables has the 
form $\mathrm{Bo}=\tilde{\rho} \tilde{H}^{2} \tilde{g} \cos \alpha /(k \tilde{\sigma})$. Here, the dimensionless parameter is $k=P_{\mathrm{cr}} \Delta t^{2} /\left(\rho_{\mathrm{cr}} h^{2}\right)$. The reduced values $\tilde{\rho}=1.93, \tilde{g} \cos \alpha=0.000007, \tilde{\sigma}=1.8$ are chosen. The initial reduced height of droplet is $\tilde{H}=\widetilde{R}_{0} \approx 115$. Hence, the initial value of the Bond number is $\mathrm{Bo} \approx 10$. At the final stage of simulations (Fig. 1,b), the Bond number is $\mathrm{Bo} \approx 1$.

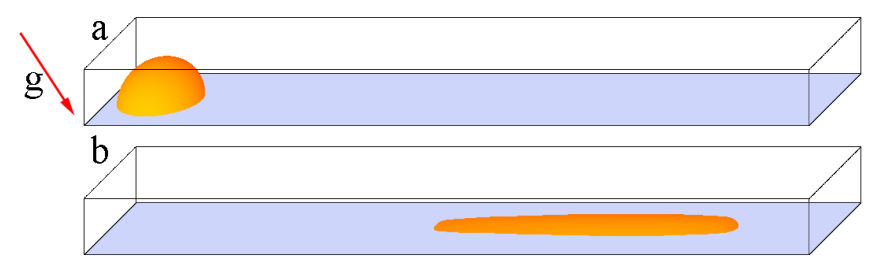

Figure 1. Droplet flowing down the inclined surface $\left(\alpha=40^{\circ}\right) . \tilde{R}_{0}=115$. Grid $2048 \times 368 \times 160$.

The second parameter $W$ is the ratio of gravity force to viscous forces $W=\mathrm{Ga} / \mathrm{Re}=\tilde{g} \sin \alpha \tilde{H}^{2} /(\tilde{v} \tilde{U})$ (the ratio of the Galilei number to the Reynolds number). Here, $\tilde{v}$ is the dimensionless kinematic viscosity, $\tilde{U}$ is the dimensionless characteristic velocity of the liquid along the surface.

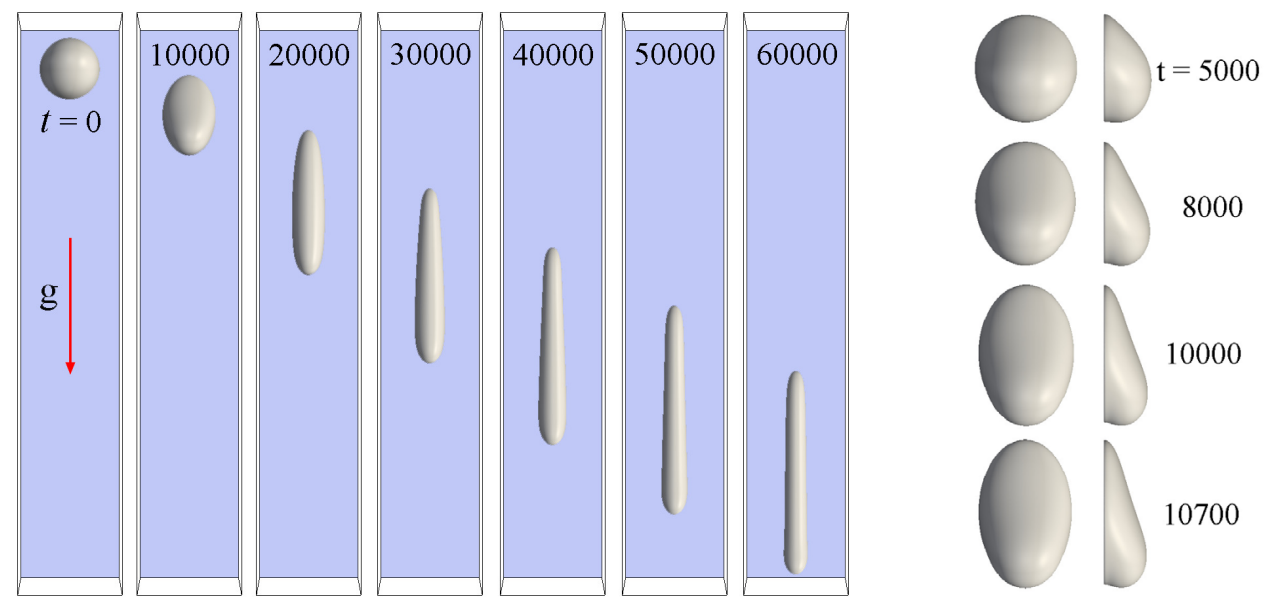

Figure 2. Droplet flowing down the vertical wall in gravity. $\tilde{R}_{0}=115$. Grid $368 \times 2048 \times 160$.

The results of three-dimensional computer simulations for the droplet flowing down the vertical wall in gravity are shown in Fig. 2. The initial spreading of the droplet on the surface is determined by the Bond number. The steady velocity of the droplet is determined by the parameter $W=\mathrm{Ga} / \mathrm{Re}$. The initial value is $W \approx 15$. The final value is $W \approx 3$ at $t=60000$. Hence, the flow tends to the quasi-stationary velocity of the droplet.

\section{Dynamic contact angles}

The dependence of a contact angle on a velocity of contact line was studied for a smooth surface. A liquid droplet is placed on the horizontal solid surface $(B=0.4)$. Then liquid is removed slowly $\left(d m / d t \approx-180 \rho_{\mathrm{cr}} h^{3} / \Delta t\right)$ from the spherical central region of the droplet as shown in Fig. 3a. Hence, the volume of droplet is reduced (Fig. 3a). We obtain the values of receding contact angle $\alpha$ vs. velocity of contact line $\tilde{V}$ (Fig. 3b). 

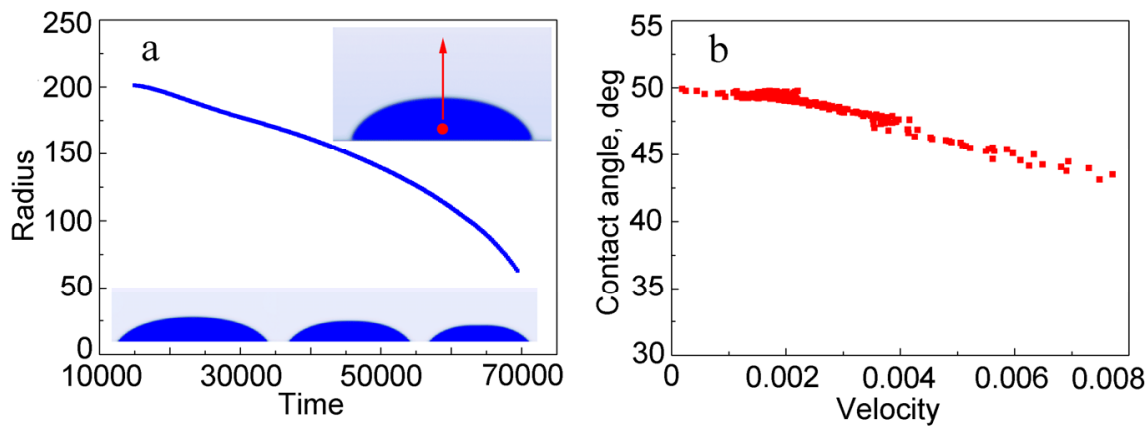

Figure 3. Reduced radius of contact line vs. time (a). Receding contact angle vs. velocity of contact line (b). Slow regime.

\section{A rupture of the thin liquid films}

The simulations of a rupture of thin liquid films on the nonwettable solid substrate due to the thermocapillary effect (Marangoni effect) were also carried out. For the prescribed axisymmetric temperature distribution $T=T(r)$ with a flattened vertex, a diverging flow in the film in the vicinity of the hot spot is generated because of the gradients of the surface tension along the film (Fig. 4a-f). The rupture of the liquid film occurs not in the center of symmetry but along a certain circle where the gradient of the temperature is more pronounced [6]. As a result, a central liquid disk is formed (Fig. $4 a, b)$. The disk transforms initially into the toroidal figure due to surface tension (Fig. 4c,d) and then into an oscillating droplet (Fig. 4e,f). The rupture of free-hanging thin film for the non-axisymmetric temperature distribution $T=T(x, y)$ is shown in Fig. $4 \mathrm{~g}$.

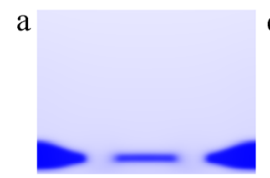

b

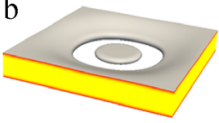

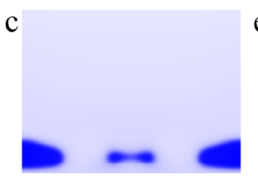

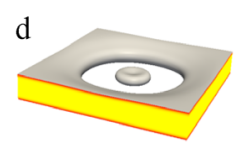

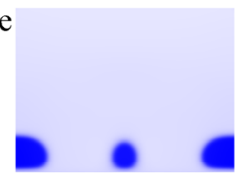

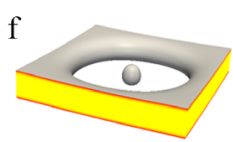

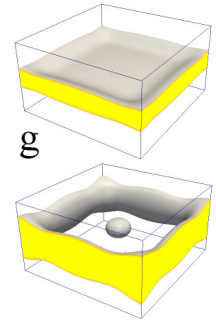

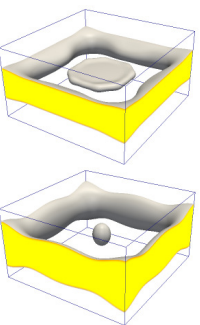

Figure 4. Thin film on nonwettable solid substrate (a-f). Free-hanging thin film at prescribed non-axisymmetric temperature distribution (g). Periodic boundary conditions are used along the $x$ and $y$ directions.

\section{References}

1. Y.H. Qian, D. d'Humières, P. Lallemand, Europhys. Lett., 17, 479-484 (1992)

2. A.L. Kupershtokh, Computers and Mathematics with Applications, 59 2236-2245 (2010)

3. X. Shan, H. Chen, Phys. Rev. E, 47 1815-1819 (1993)

4. Y.H. Qian, S. Chen, Int. J. Mod. Phys. C, $8763-771$ (1997)

5. A.L. Kupershtokh, D.A. Medvedev, D.I. Karpov, Computers and Mathematics with Applications, 58 965-974 (2009)

6. A.L. Kupershtokh, E.V. Ermanyuk, N.V. Gavrilov, Comm. Comp. Phys., 17 1301-1319 (2015) 